Pedagogía y Saberes No. 50

Universidad Pedagógica Nacional

Facultad de Educación. 2019. pp. 97-108

\title{
Riquezas y límites del enfoque por "competencias" del ejercicio de la profesión docente hoy
}

Traducción

Richesses et limites de l'approche par «compétences » de l'exercice du métier d'enseignant aujourd'hui

Resources and Limits of the "Competence" Approach from Today's Teaching Profession Excercise

Philippe Meirieu*

Traducido por: Paula Alexandra Montenegro Gómez**

Para citar este artículo:

Meirieu, P. (2019). Riquezas y límites del enfoque por “competencias" del ejercicio de la profesión docente hoy (Trad. P. Montenegro). Pedagogía y Saberes, 50, 97-108.

* Profesor emérito de la Universidad Lumière-Lyon 2 (France).

Correo electrónico: philippe.meirieu@orange.fr

** Profesional en Filología e Idiomas - Francés de la Universidad Nacional de Colombia.

Correo electrónico: paamontenegrogo@unal.edu.co Código oRCID: orcid.org/0000-0003-4571-8502 


\title{
Resumen
}

Este artículo de reflexión cuestiona uno de los "lugares comunes" que orientan hoy la formación de profesores: "Enseñar, educar sería en primer lugar, y, antes que nada, y tal vez exclusivamente, una cuestión de competencias por adquirir". No obstante, el cuestionamiento no consiste en un rechazo al concepto de competencia, como suele suceder desde algunas perspectivas que se declaran críticas, sino en someter el concepto a un análisis riguroso, según el cual el trabajo pedagógico es irreductible a la acumulación de saber-hacer y a la práctica de ejercicios mecánicos, pues se refiere a la capacidad de inventar situaciones generadoras de sentido que articulan estrechamente el descubrimiento y la formalización, es decir, que hacen referencia a la capacidad de pensar.

\section{Palabras clave}

competencia; trabajo pedagógico; formación de profesores

\section{Résumé}

Cet article de réflexion interroge l'un des points communs qui guident aujourd'hui la formation des enseignants : "Enseigner, éduquer serait d'abord et avant tout, et peut-être exclusivement, une question de compétences à acquérir". Cependant, le questionnement ne consiste pas à rejeter le concept de compétence, comme c'est généralement le cas dans certaines perspectives déclarées critiques, mais à soumettre le concept à une analyse rigoureuse, selon laquelle le travail pédagogique est irréductible à l'accumulation de savoir-faire et à la pratique des exercices mécaniques, puisqu'il renvoie à la capacité d'inventer des situations qui génèrent des sens qui articulent étroitement découverte et formalisation, c'est-à-dire qui font référence à la capacité à penser.

\section{Mots-clés}

compétence; travail pédagogique; formation des enseignants

\begin{abstract}
This reflection article questions one of the "common places"; that guide teacher training today: "Teaching, educating would be first and foremost, and perhaps exclusively, a question of skills to be acquired". However, the questioning does not consist in a rejection of the concept of competence, as is usually the case from some perspectives that are declared critical, but in subjecting the concept to a rigorous analysis, according to which pedagogical work is irreducible to the accumulation of know-how and the practice of mechanical exercises, since it refers to the capacity to invent situations that generate meaning that closely articulate discovery and formalization, that is, that refer to the capacity to think.
\end{abstract}

\section{Keywords}

competence; pedagogical work; teacher training 


\section{Introducción: La reflexión pedagógica en lucha contra los "lugares comunes"}

A pesar de algunas treguas mediáticas donde sus adversarios parecen debilitarse, los pedagogos

siempre han tenido la impresión de estar "en el frente". Y lo están, en efecto, permanentemente. Lo están, en primer lugar, porque deben recordar incesantemente -empezando por ellos mismosque nadie está destinado al fracaso ni condenado a la exclusión, que cada uno puede aprender y crecer, que la transmisión de la cultura no puede tener por objetivo seleccionar a las élites, sino que debe permitir a todos acceder al placer de pensar y al poder de actuar. Contra todas las formas de fatalismo y renuncia, contra la obsesión clasificadora de nuestras sociedades, contra el encierro sistemático de los sujetos en "rejillas" por parte de las instituciones que se conforman con "gestionar el flujo", ellos son portadores de una "insurrección fundacional" que los pone siempre, más o menos, en posición de combate y los hace parecer "aguafiestas de la enseñanza".

Pero los pedagogos están también "en el frente" porque propenden al mismo tiempo - y eso es lo que molesta de ellos- por el "respeto" del alumno. Si suponen su educabilidad, también recuerdan sin parar que la educación no es una fabricación y que nadie puede aprender y crecer por nadie más. Si bien concuerdan con que existe una anterioridad de la cultura y de los saberes sobre los sujetos que vienen al mundo, no dejan de afirmar que cada sujeto debe apropiarse de esa cultura y esos saberes en un proceso singular que requiere su compromiso personal. Ontológicamente, el saber precede al aprendizaje; pedagógicamente, el sujeto precede al saber, y es esta doble antecedencia la que hace de ésta una empresa difícil. Es ella la que hace parecer a los pedagogos "aguafiestas de la simplificación".

Al mismo tiempo que consideramos que nunca se terminan de proponer recursos y métodos para volver los saberes atractivos y accesibles, aceptamos su impotencia sobre un sujeto que solo puede decidir aprender y crecer, por lo que es necesario luchar a diario contra todas las tentaciones del pensamiento mágico. Hay que rechazar la devoción hacia los programas que no requieren más que decretar su importancia para garantizar su adquisición. Es necesario renunciar a la creencia del poder irresistible del mandato de aprender, repetido continuamente por todos los actores de la escuela y transmitida en eco por las familias y los medios. Es necesario abandonar tanto la nostalgia de un pasado idealizado donde "los alumnos aún estaban motivados y eran trabajadores" como la utopía de una sociedad ideal donde por fin las tecnologías digitales permitirían a todos los niños estar milagrosamente al mismo nivel con los saberes e intercambiarlos espontáneamente en una gran fraternidad planetaria... La pedagogía sabe que aprender no es fácil, que enseñar requiere, al mismo tiempo, mucha ambición y modestia. Es por eso que los pedagogos también pueden enorgullecerse de parecer a veces "aguafiestas de la estupidez".

Para ser convincentes en su proyecto, los pedagogos deben, evidentemente, dar ejemplo del rigor que pretenden encarnar. Es por eso que nunca deben descuidar el cincelado del concepto en favor de lo conmovedor del discurso. Legítimamente indignado por la terrible constatación de que "la especie es malvada con sus niños" (Hameline, 2000, p. 95), el pedagogo con frecuencia se deja llevar por el arrebato militante sin siquiera guardarse las espaldas. Es así como practica gustosamente la denuncia sistemática y la recuperación moral. El "respeto del niño" se convierte para él en un estandarte cómodo que permite reclutar médicos, psicólogos y sociólogos, padres, maestros y trabajadores sociales, todos unidos tras algunas fórmulas ampliamente consensuadas: "el niño es un ser humano con todas las de la ley", "hay que respetar las leyes de su desarrollo", "debemos escuchar su palabra, responder a sus necesidades y a sus intereses", "solo puede aprender si es activo y colaborando con los demás", etc. Todas estas afirmaciones parecen constituir un cuerpo de doctrina bastante estable que permite distinguir y promover las "buenas prácticas" educativas. Éstas funcionan como un signo de reconocimiento para todos aquellos que, de una manera u otra, militan en "la causa de los niños".

Probablemente no podemos escapar por completo de esta vulgata. Es incluso necesaria para alimentar el "foco mitológico" donde se origina nuestra capacidad de afrontar diariamente la multitud de pequeños y grandes fracasos como las persecuciones puntilludas de las jerarquías e instituciones. Para volver al trabajo y afrontar la inevitable petulancia de los especialistas del "No es posible, iya se los había dicho!", es necesario un valor que los análisis filosóficos más minuciosos y los trabajos científicos más rigurosos son incapaces de comunicarnos. También necesitamos palabras rituales y colectivos convencidos, certezas proclamadas y recordatorios de nuestros "valores fundacionales". Ya que el ser humano no vive solamente de ciencia y aquel que todas las mañanas lleva a cuestas niños molestos, absortos o simplemente indiferentes hacia lo que estamos encargados de transmitirles, no puede pres- 
cindir de algunos "lugares comunes" pedagógicos para enfrentar al océano de indiferencia tecnocrática de su institución. ${ }^{1}$

Sería pretencioso entonces aquel o aquella que proclamara: "Lugares comunes pedagógicos, de esta agua no beberé...", pero sería ingenuo aquel o aquella que imaginase que esos lugares comunes van a permitirle ganar la batalla contra la fatalidad y la injusticia, por la democratización del acceso a los saberes y la emancipación de los pequeños a su cargo. Lo que nos mueve no nos permite combatir verdaderamente al adversario, al contrario, al exponerlo sin precaución se corre el riesgo del ridículo y el desprestigio. Las profesiones de fe, en sus aproximaciones inevitables y su entusiasmo ingenuo, suscitan siempre una mezcla de ternura y de lástima... iy éstas apenas si arman la mano del militante!

Es así como eso que usualmente llamamos "la pedagogía" puede parecer un revoltijo ideológico donde los acrónimos nacidos del movimiento de la escuela nueva, estructurado a principios del siglo $\mathrm{xx}$, permiten todas las interpretaciones y abren la puerta a todas las suspicacias:

\begin{abstract}
¿"El alumno al centro" no es el niño-rey? ¿"El niño activo" no es la totemización del bricolaje en detrimento de los saberes elaborados? ¿El llamado a la "motivación" no es el capricho institucionalizado? ¿La "individualización” no es individualismo? Y, ¿la "personalización" no es encerrarse en lo dado? En cuanto a la "pedagogía de proyectos", ¿no confunde la escuela, que está hecha para aprender, con el taller o la fábrica, que están hechos para producir? (Meirieu, 2013, p. 5) ${ }^{2}$
\end{abstract}

Es por esto que, sin negar la necesidad de una cierta retórica pedagógica, el pedagogo debe obligarse regularmente a hacer sus lugares comunes a prueba del concepto. Le corresponde escudriñar con rigor informado las "evidencias compartidas" sin complacencia, pero sin concesión para disipar, tanto como se pueda, los malentendidos, aunque tampoco sin ilusión: los malentendidos nunca se erradican definitivamente. Otros emergen donde se creía haber aclarado las cosas, en los repliegues mismos de la

1 Daniel Hameline propone una teoría de los "lugares comunes pedagógicos" a la que me refiero: en el "lugar común", explica, "la argumentación se plantea entre el truismo y la evidencia" (1986, p. 63); y "es el eslogan mismo el que, a costa del malentendido latente, permite la unidad de acción” (1986, p. 64).

2 Sobre esta historia, ver mi obra Pédagogie: des lieux communs aux concepts clés. tentativa de aclaración. Otros, incluso, resisten o se desplazan. Así, la tarea jamás se termina, jafortunadamente! Si no tuviésemos más malentendidos por aclarar, ya no tendríamos mayor cosa por decirnos.

Es por eso que les propongo dedicarme a un lugar común entre otros, un lugar común que hoy ha invadido casi totalmente el mundo de la formación de los docentes, un lugar común que a duras penas nos atrevemos a cuestionar y que, sin embargo, voy a cuestionar frente a ustedes: "Enseñar, educar sería en primer lugar, $y$, antes que nada, $y$ tal vez exclusivamente, una cuestión de competencias por adquirir".

En efecto, en educación siempre hay que desconfiar de las palabras y las expresiones que tienen mucho éxito y se convierten fácilmente en "lugares comunes", mucho más cuando se trata de una palabra como "competencia", vastamente utilizada para administrar de manera tecnocrática la cuestión de la "empleabilidad" en el "mercado" del trabajo. Más aún cuando, como lo mostró Gaston Bachelard en el plano epistemológico, es deconstruyendo los "lugares comunes" que hacemos que avance el pensamiento y progrese la investigación.

Soy consciente de ceder de esta manera, por una parte, a una "subversión escandalosa", pero es una subversión a la que nos invitaban, desde 1945, los "maestros revolucionarios" que habían contribuido a elaborar los principales conceptos de la escuela nueva:

No asuman nunca estas pistas y estas luces como definitivas, no reestablezcan los tabúes, no tracen con la rutina los nuevos caminos. Lo que es escandaloso no es que los educadores critiquen y busquen mejorar los métodos de Mme. Montessori, de Ferrière, de Decroly, de Piaget, de Washburne, de Dottrens o de Freinet. El escándalo educativo es que de nuevo haya "fieles" que pretendan erigir en el mismo lugar donde estos educadores se detuvieron, capillas guardianas celosas de las nuevas tablas de la ley y de las reglas magistrales, y que no se comprenda que el pensamiento de Ferrière, de Piaget, de Washbourne, de Dottrens o Freinet es, esencialmente, cambiante, que hoy no es lo que fue hace diez años y lo que, en diez años, las nuevas generaciones habrán hecho germinar. (Freinet, 1945, p. 25). 


\section{Enseñar hoy: nuevos desafíos por asumir}

Sin entrar en detalles que, por otro lado, exigirían ser ampliamente matizados en función de las situaciones naciones y sociales muy diversas de la modernidad, quisiera evocar brevemente cómo el paradigma tradicional de la enseñanza ha fracasado y necesita la introducción de nuevos enfoques:

1. Enseñar fue por mucho tiempo - y con justa razón- considerado como "la capacidad de llevar el saber al más alto grado de inteligibilidad para el otro" ... esto con el fin de volver el saber accesible a la inteligencia del otro y, simultáneamente, contribuir a estructurarla.

2. Este proyecto siempre chocó con resistencias ${ }^{3}$, pero en tanto que esas resistencias eran consideradas parte inevitable del fracaso y toleradas tanto desde el punto de vista social como político... este paradigma ("llevar el saber al más alto grado de inteligibilidad para el otro") se consideraba suficiente para definir la profesión docente y estructurar su formación.

3. La modernidad educativa es inaugurada por Itard quien, en 1800, quiere, contra todo pronóstico, contra toda expectativa, "educar" al "salvaje del Aveyron" (considerado entonces por todos los expertos como un "estúpido por naturaleza") y por Pestalozzi que, en 1799, intentará instruir a los huérfanos de Stans que, sin embargo, lo rechazaron violentamente. Esta modernidad educativa hace del postulado de la educabilidad el principio pedagógico por excelencia. Sin quitarle nada al proyecto de la inteligibilidad de los saberes (que evidentemente guarda un poder heurístico esencial), ésta vuelve a poner en cuestión el "principio de la inteligibilidad suficiente". No basta con que un saber sea perfectamente dominado e inteligible por el maestro para que sea transmitido.

4. Nuestro proyecto de transmitir choca, en efecto, con la resistencia... de aquellos que parecen no poder aprender... de aquellos que no quieren aprender... de aquellos que quieren saber sin aprender... de aquella cuya "relación con el saber" no es tenida en cuenta por la institución escolar.

3 Lo que llamé en mis trabajos "el momento pedagógico". (Meirieu, 1995).
5. Esto máxime considerando que ya no hay una superposición entre el proyecto de la institución y el de cada uno de sus actores en su singularidad.

6. En estas condiciones, frente a esta resistencia, si nos guía el postulado de la educabilidad, debemos escapar de la doble tentación: la del abandono fatalista, por un lado, y la de imponerse por la fuerza, por el otro.

7. Así, hemos sido destinados a construir una nueva profesionalidad fundada sobre la inventiva pedagógica. Si el profesor debe "convencer sin vencer" e "inspeccionar" sin adiestramiento ni violencia, necesita competencias pedagógicas: "saber actuar", movilizando recursos para alcanzar sus objetivos en contextos particulares.

\section{Competencias necesarias para asumir los retos de enseñar hoy}

Existe una multitud de "listas de competencias" que permiten llenar un "cuadro de indicadores" a partir del cual formar a los profesores en el ejercicio de su profesión. Aquí presento uno, discutible como todos los demás, pero que es bastante representativo de lo que se utiliza en los centros de formación.

Para poder enseñar eficazmente hoy, un profesor debe ser capaz de:

1. Concebir y organizar situaciones de aprendizaje (identificar el objetivo, la tarea, el obstáculo, las imposiciones y los recursos en función de la operación mental por efectuar).

2. Animar las situaciones de aprendizaje e intervenir para mantener el compromiso de cada alumno.

3. Ayudar a formalizar los aprendizajes efectuados: permitir a cada alumno observar lo que ha aprendido a través de lo que ha hecho.

4. Articular las situaciones de aprendizaje para que constituyan una progresión coherente y perceptible para el sujeto.

5. Hacer uso de los recursos materiales, documentales y tecnológicos necesarios.

6. Documentarse y trabajar en equipo pedagógico. 
7. Participar en la gestión de la escuela.

8. Informar e implicar a los padres.

Formar a un profesor que se apega a cada una de estas competencias permite...

- Librarse de una visión arcaica del innatismo profesional.

- No refugiarse en un inefable que esconde las complicidades culturales.

- No confundir los objetivos con los prerrequisitos.

- Evitar los implícitos pedagógicos.

- Identificar los diferentes campos de acción posible de la profesión.

- Construir currículos (pasar de una lógica de enseñanza donde nos conformamos con yuxtaponer las enseñanzas a una lógica de formación donde trabajemos con la persona en términos de desarrollo profesional).

- Pensar y poner en marcha la transferencia de competencias (utilizar conceptos y modelos en función de los contextos)...

\section{Las ventajas de un enfoque por competencias para el profesor... y para el alumno}

La noción de competencia constituye entonces, en varios aspectos, un progreso esencial para la reflexión educativa y la formación de los maestros: ésta permite escapar de todas las formas de fatalismo y de encierro que bloquean la democratización del acceso a los saberes. Evidentemente, todo esto pone el listón muy alto: afirmar que todo sujeto puede adquirir todas las competencias posibles e imaginar medios para llegar a este fin que sean compatibles con el respeto de su libertad, no es una tarea sencilla. Probablemente sea más una "utopía de referencia" que un objetivo a corto plazo. Más un "principio regulador" que un "principio constitutivo", según la distinción elaborada por Kant4: un poco como "la belleza" para el artista... Nadie puede pretender haberla alcanzado completamente ya que, entonces, no se la buscaría.

4 Kant (trad. en 1968) distingue los "principios constitutivos", que son objetos de certidumbre, de los "principios reguladores", que sostienen el esfuerzo del pensamiento, le prohíben satisfacerse con soluciones provisionales, le impiden esperar una posible finalización de su proyecto, sin desmovilizarla por ese motivo.
Ahora bien, todo el mundo sigue buscándola. ¡Y es esta búsqueda, precisamente, la que se pone manos a la obra en toda la historia del arte!

Por otra parte, muy lejos de los referentes conductistas a las que la llevamos a veces, la noción de competencia constituye un antídoto precioso al primado de la tarea que amenaza sistemáticamente la pedagogía escolar y la formación de los maestros. El imperialismo de la tarea es permanente y es necesario liberarse de él con tanta fuerza y determinación que nos despeguemos de la fatalidad y de la "psicología de los dones". La tarea es aquello sobre lo que el sujeto se moviliza, lo que ve, que guarda en la memoria o en sus archivos. La tarea es la situación de aprendizaje, el deber de francés, el ejercicio de matemáticas - con su "resultado", correcto o equivocado-, el dibujo, la experiencia, el partido, la secuencia de gimnasia, el muro de escalada, etc. La tarea es lo que el niño debe "hacer" y lo que el profesor le debe "hacer hacer". Debe hacerla porque ya la ha visto, tiene una representación mental de ésta, sabe lo que se espera de él... La tarea también es lo que va a proveer, en caso de éxito, la satisfacción narcisista y, en caso de fracaso, la que va a engendrar la decepción y a contribuir a la degradación de la imagen de sí mismo.

Sin embargo, la tarea es fugaz, en extremo fugaz. Pocos alumnos guardan toda la vida la totalidad de sus cuadernos y deberes. Muy pocos guardan el recuerdo de todas las tareas que tuvieron que realizar en la escuela. Afortunadamente, por cierto: ¡Son demasiadas y no son importantes! A los veinte años, no recordamos todos los ejercicios que llevamos a cabo para aprender a leer, no conservamos la totalidad de nuestros cuadernos de primero de primaria... ¡pero sabemos leer! Lo que fue construido, en el marco de esta multitud de tareas olvidadas, fue bien conservado: se convirtió en una competencia de la que la persona se apropió completamente, que pone en práctica libremente, desde su propia iniciativa. Allí está lo esencial... y lo que hace que el aprendizaje haya sido exitoso.

Es esto, precisamente, lo que distingue a un sistema de formación de un sistema de producción. En la fábrica, el taller o la oficina de gestión de proyectos, se producen bienes materiales o culturales. La escuela, en el sentido estricto, no "produce" nada; forma. Permite a los individuos construir competencias y, al mismo tiempo e indisociablemente, adquirir el gusto por saber, el sentido del análisis y el pensamiento crítico. Todas éstas son cosas que, precisamente, no son puestas en circulación al salir de clase, en el sistema de producción; cosas que incrementan, en realidad, habilidades mentales invisibles: invisibles porque 
la observación directa no permite constatar su existencia sin lugar a duda; invisibles porque algunas pueden no revelarse hasta mucho tiempo después y de manera totalmente imprevisible.

Nos cuesta acostumbrarnos, ya que este vuelco propio de la escuela altera todas las representaciones de la vida cotidiana: un artista, un contramaestre, un agricultor o un tipógrafo deben invertir toda su energía en "la tarea". Es a partir de ella que serán juzgados. Un alumno no puede ser juzgado por sus producciones... 0 entonces, no entendemos por qué sus profesores le prohíben "copiar" o se inquietan cuando consigue realizar una tarea por casualidad, por un golpe de suerte que no se reproducirá nunca. No vemos por qué deberíamos interesarnos tanto -en realidad, mucho más- en la manera en la que el alumno llega al borde de la piscina o a la cima del muro de escalada como en el hecho de llegar lo más rápido posible... Además, en el circuito productivo, alguien que se encuentra con una dificultad debe tener la sabiduría de renunciar y de dirigirse a alguien más competente que él. En la institución escolar, esto está prohibido, o es tolerado si y solo si la persona más competente ayuda a aquella que está en dificultades a encontrar ella misma la solución o, al menos, a progresar en su comprensión de los fenómenos, en su capacidad de superar los obstáculos. En el circuito productivo, incluso si los individuos continúan perfeccionándose a lo largo de su carrera, sus competencias existen previamente a sus acciones. En la escuela, es la acción de aprender la que permite adquirir las competencias.

Y puesto que el objetivo está constituido por competencias, las actuaciones de los alumnos no pueden considerarse más que indicadores, inevitablemente aproximativos. Los lingüistas señalan que una "competencia lingüística" nunca garantiza la "actuación lingüística" ... y viceversa: un sujeto puede saber hablar y no conseguir hacerlo en condiciones determinadas; al contrario, un sujeto puede producir una actuación por casualidad y sin ser capaz de reproducirla.

Así comprendemos el estado paradójico de todo ejercicio y toda evaluación escolar: éstos exigen la realización de una actuación para poder inferir de allí la existencia de una competencia. Focalizan la atención de aquél que debe aprender sobre una tarea, mientras que lo que se busca es una habilidad estabilizada que, precisamente, éste no puede comprender antes de haberla adquirido. Obligan a hacer aquello que, en realidad, no tiene importancia... sin poder nombrar lo verdaderamente importante.
El sujeto ya no debe situar su satisfacción en la realización de la tarea, sino en el progreso que la superación del obstáculo le permitirá hacer. Ahora bien, hacer esta ruptura no es para nada fácil. Es incluso, en muchos aspectos, contra natura o, más exactamente, va en contra de todas las costumbres adquiridas fuera de la escuela. Es así que aquí el rol del maestro es esencial: debe, de alguna manera, impedir que el alumno pueda "tener éxito" sin "comprender". Es en primer lugar una cuestión del sistema, pero todo sistema es estéril si el maestro no acompaña al alumno en un cambio esencial de postura: aceptar situar su placer narcisista en el "comprender" y no en el "tener éxito"; desplazamiento fundamental que un niño puede efectuar solamente si encuentra a un adulto que dé testimonio de las satisfacciones posibles que este desplazamiento conlleva. Vemos entonces que no es solamente una cuestión del sistema.

Nunca insistiremos lo suficiente sobre la distinción esencial, constitutiva de la institución escolar, entre la tarea y la competencia: un profesor no puede hacer clase sin enunciar claramente las instrucciones concernientes a las tareas por cumplir, pero no puede "faire aile l'École" sin diseñar y evaluar las competencias que los alumnos deben dominar. La distinción entre "tarea" y "competencia", la desintricación permanente entre estas dos realidades es, como tal, un objeto de trabajo esencial, al mismo tiempo de la formación de los profesores y de la práctica cotidiana en la clase con los alumnos.

Es necesario insistir en el hecho de que la competencia es una herramienta valiosa para pensar la transferencia. Si la escuela solo enseñara a tener éxito en clase, ésta claramente constituiría una inversión considerable para un interés en extremo mínimo. En efecto, en la escuela se deben aprender cosas que podrán ser reutilizadas afuera, en otro contexto. Es por esto que es mucho más importante "saber que se sabe" que "saber", ya que aquél que sabe sin saber que sabe debe esperar a que se le pida restituir los que aprendió para utilizarlo. Aquél que "sabe que sabe", por otro lado, no depende de la interrogación del maestro: puede tomar la iniciativa de poner sus competencias en acción...

Aun así, también es necesario que las utilice acertadamente, que la competencia que construyó sea utilizada en una familia de situaciones en la que resulte pertinente y eficaz. Una competencia es una llave que no funciona indiferentemente en todas las cerraduras. Es así, por ejemplo, para la competencia que puede tener un conductor de un automóvil para tomar la información de su ambiente visual y sonoro 
con el objetivo de ajustar su velocidad y dominar su dirección. Uno podría pensar que esta competencia es la misma que la del conductor de un barco, la del ciclista o incluso la del esquiador. Este último, en efecto, debe hacerse a la información en tiempo real sobre el estado de la nieve y de la pendiente y repercutirlas en decisiones inmediatas en el movimiento de su cuerpo para controlar mejor su descenso. Sin embargo, al ver de cerca, no puede tratarse de una especie de "competencia general" - lo que a veces llamamos una "capacidad" - que se formularía de manera transversal, independientemente de los contenidos que ponen en práctica: "tomar información de su ambiente para ajustar su trayectoria". La información necesaria para conducir o para esquiar no le compete al mismo corpus de conocimientos y, si hay una proximidad formal entre los procedimientos, es a causa de un acercamiento a posteriori. Como lo demostró Vygotsky (1985, p. 111), es imposible aprender esta capacidad "en abstracto", independientemente de todo contenido, de toda situación precisa... y "vestirla" con diferentes contenidos.

La noción de competencia, bien utilizada, permite pensar en el difícil problema de la transferencia de conocimientos. Construir una competencia es, precisamente, volverse capaz de resolver un problema dado en una situación dada. Puesto que hemos analizado la situación, podemos reaccionar de manera pertinente, y ya que hemos sido capaces de hacer este análisis, también nos volvemos capaces de utilizar esta competencia en otras situaciones en las que resultará pertinente. La competencia sigue siendo local, pero puede ser utilizada de manera acertada en otros lugares. Es su nivel de experticia lo que garantiza su transferibilidad. Cuanto más soy experto en una competencia específica; es decir, cuanto más comprendo lo que hago construyendo esta competencia, me vuelvo más competente... y así, me vuelvo capaz de construir nuevas competencias. Este "efecto bola de nieve" da la sensación de que las adquisiciones en un campo pueden repercutir en otro...

\section{Los límites de la noción de "competencia" para pensar la profesión docente y formar}

Por supuesto, el primer peligro inherente al uso sistemático de la noción de "competencia" es el de olvidar el principio de "inteligibilidad necesaria", pero también podemos:
- Olvidar el objetivo del proyecto educativo (educar y no fabricar).

- Olvidar los aportes inestimables de la historia de la pedagogía.

- Ignorar que muchas cosas se desarrollan con microgestos, en la urgencia y la incertidumbre.

- Dejar que se extinga la inventiva, creyendo que todo está en las "competencias" y que basta con comprender los problemas para obtener las soluciones.

- Caminar hacia la aniquilación, la proletarización y la pérdida de identidad de la profesión.

- Cambiar radicalmente de la lógica de la institución (construida sobre valores) a la lógica de servicio (para satisfacer los intereses individuales).

Sobretodo, no hay que olvidar que, de la misma manera que ninguna profesión se reduce a la suma de las competencias necesarias para ejercerla, ningún saber se reduce a la suma de las competencias necesarias para dominarlo. ¿Bastan las competencias gráficas, escriturales, ortográficas y gramaticales para ingresar a una cultura letrada? No lo creo, ya que entrar en lo escrito es ser capaz de transformar las ataduras de la lengua en recursos para el pensamiento. Es ser capaz de entrar en una "obra" y, a su vez, de crear una.

Este juego de imposiciones y recursos se enmarca en un trabajo pedagógico irreductible a la acumulación de saber-hacer y a la práctica de ejercicios mecánicos. Hace referencia a la capacidad de inventar situaciones generadoras de sentido que articulan de manera estrecha el descubrimiento y la formalización. Ahora bien, en algunos países hoy nos alejamos de esto a grandes pasos, con libretas de competencias yuxtaponen competencias tan diferentes como "saber dar cuenta de la creatividad" y "saber adjuntar un documento a un correo". Entonces, ¿qué puede significar que "el alumno tenga el $60 \%$ de las competencias requeridas"? La noción de competencia hace referencia tanto a saberes técnicos reproducibles como a capacidades demostrables que a nadie interesa saber cómo se forman. Estos marcos de referencia aniquilan la noción misma de cultura y hacen perder de vista la formación hacia la capacidad esencial a mis ojos: la capacidad de pensar. 


\section{Nunca perder de vista los objetivos y siempre volver a éstos: ¿qué vale la pena enseñar hoy? Lo que une y lo que libera...}

Frente al ascenso de todas las desviaciones de la modernidad, la respuesta que daba Olivier Reboul, hace cuarenta años, a la pregunta " ¿Qué es lo que debe fundar la educación?" (Reboul, 1989) es más actual que nunca: "Lo que une y lo que libera". En efecto, tenemos al mismo tiempo necesidad de unidad —de lo común sin comunitarismo-y de libertad - de individuos sin individualismo- - Necesitamos redescubrirnos parecidos y encontrar la fuerza de afirmarnos diferentes.

Sabemos que la escuela ha sido vastamente construida sobre la convicción de que los saberes unen mientras que las creencias separan. Evidentemente, ésta sigue siendo fundacional: como son validados y reconocidos, los saberes nos congregan y, aunque no erradican automáticamente las creencias, estabilizan las relaciones de los humanos sobre bases que les permiten no precipitarse los unos sobre los otros para destruirse. Compartir saberes es aceptar una verdad común que puede permitir confrontar las creencias sin agredirse, pero es necesario que el maestro que invita al alumno a distinguir los saberes de sus creencias no enseñe sus propios saberes como creencias. Viejo desafío pedagógico del que Jules Ferry era particularmente consciente, él que invitaba a los instructores a inspirarse con los "métodos activos" de Pestalozzi (Buisson, 2016). Objetivo de todos los días, en todas las clases, sobre el más mínimo aprendizaje propuesto: "Lo que digo no es una opinión... En la escuela no nos justificamos a nosotros mismos, sino que justificamos... y nunca es el que grita más fuerte o el que seduce más quien tiene razón, sino aquél que lo demuestre mejor" (s. p.). Propósito esencial, sobre todo porque la experiencia común vendrá a mediar entre las posiciones de unos y otros.

Queda el hecho de que los estudiantes no se reducen solamente a su actividad cognitiva: son seres que viven de inquietudes y esperanzas, de preguntas que los atormentan y de pasiones que los animan. La tradición cartesiana, aunque permita hacer de "la interargumentación racional" el principio regulador del debate público, no puede decretar la abolición de esa vía psíquica y caótica. La cultura puede, en cambio, darle forma y hacer de ella un medio de encuentro entre los seres. El arte, la literatura y la filosofía nos permiten, en efecto, descubrirnos hijos e hijas de las mismas preguntas fundacionales. Y si no compartimos las mismas respuestas, al menos nos reconocemos a través de nuestros interrogantes como depositarios, en conjunto, de la "humana condición”. ¿Qué nos enseña la figura del ogro en los cuentos de nuestra infancia, sino que es difícil amar a alguien sin devorarlo? Y aquél que pretendiera poseer la solución definitiva para asociar milagrosamente el amor a la libertad, evidentemente sería un impostor. A una pregunta común, respuestas particulares y la posibilidad de confrontar nuestras posiciones recíprocas sin excluirse unos a otros del círculo de lo humano. El encuentro con la literatura y las Humanidades permite la confrontación con lo común de los individuos... y por allí pasa el aprendizaje de la empatía, que Martha Nussbaum muestra bien como la "pasión democrática" por excelencia (Nussbaum, 2011).

Así, la escuela puede crear lo común: puede hacerlo por la manera en la que transmite los saberes; puede hacerlo al promover las actividades artísticas y culturales; puede hacerlo al practicar una pedagogía cooperativa donde la implicación de cada una y cada uno permita el éxito de todos. Puede hacerlo también al hacer de la dimensión ecológica de los saberes un elemento estructurante del descubrimiento de la solidaridad profunda que nos une en el seno de la "Tierra Patria", como dice Edgar Morin (2010). Lo común se construye en la escuela, ya que, en nuestra sociedad republicana, la escuela no es simplemente el lugar donde cada uno aprende; es el lugar donde aprendemos juntos y, con ese aprendizaje, aprendemos a hacer sociedad.

Sin embargo, si la educación es descubrimiento de aquello que une a los humanos, también es, simultáneamente, aprendizaje de lo que los libera: aquello que libera del egocentrismo inicial y de la inmediatez de la pulsión, lo que libera del fantasma de la omnipotencia y de la sumisión ciega al poder, lo que libera de los prejuicios y estereotipos, lo que libera de todos los encierros y, en particular, de la influencia de los gurús que proveen identidad y seguridad al precio terrible de la abdicación de toda libertad.

Para ello, la escuela debe asumirse deliberadamente como un espacio de desaceleración. Lejos de la recompensa a la respuesta rápida, debe promover la reflexividad crítica. Debe aplazar la pulsión y la respuesta rápida inmediata para sacar provecho del tiempo para anticipar, intercambiar, documentarse, reflexionar... en fin, de aprender a pensar. Estamos lejos de ello, nosotros que corremos siempre en los pasillos y tras los programas, que huimos del silencio como de la peste y calificamos un deber de forma 
definitiva, sin dar al alumno la posibilidad de aprovechar nuestros consejos para mejorarlo. Frente a la inmediatez del "todo y de inmediato" que la maquinaria publicitaria y tecnológica promueve sistemáticamente, la escuela debe asumir deliberadamente un rol termostático. Ni rechazo brutal a la reacción del alumno, ni aceptación demagógica de su opinión, sino una puesta en suspenso: "Tomémonos el tiempo de reflexionar al respecto...". Solo así la escuela contribuirá a enseñar a los niños y adolescentes a resistirse a las seducciones de todo tipo. Seducciones mercantiles o sectarias, y también seducciones ideológicas de todas las formas de totalitarismo.

Probablemente me consideren ingenuo: ¿cómo algo que parece "cocina pedagógica" puede luchar contra el yihadismo, su propaganda y sus redes? En materia educativa, nunca vemos realmente los resultados de nuestros actos y, a fortiori, lo que hemos contribuido a impedir. Cada uno de nuestros alumnos puede cambiar radicalmente el mundo o, al menos, debemos actuar como si así fuese.

Sin embargo, seguimos dudando: ya que el pedagogo, como todo el mundo hoy en día, se ve confrontado a una pregunta a la vez muy simple y terriblemente compleja, la pregunta que hace fracasar al mismo Sócrates en las primeras líneas de La República: ¿cómo hacerse oír de aquél que no quiere escuchar nada? 0 incluso, ¿ cómo hacer atender a razones a quien no ha escogido la razón? Pregunta vana y cotidiana, pero tan difícil que preferimos sistematizar las explicaciones patológicas antes que enfrentarlas. Ahora bien, precisamente, el radicalismo yihadista hace esta pregunta en un pasaje en última instancia, que nos vuelve a cuestionar sobre nuestro proyecto de sociedad.

En efecto, lo peor es que tenemos respuestas a esta pregunta, las respuestas de nuestros adversarios: la intimidación, el reclutamiento y el control y, también, todas las camisas de fuerza tecnológicas y químicas. Respuestas estrictamente limitadas por nuestros principios democráticos: podemos protegernos de la violencia bárbara, pero no tenemos derecho de "hacer atender a razones" a los humanos por medio de coerción sistemática. En materia de convicciones, es necesario que venzamos sin vencer. Es la fragilidad de nuestra democracia, pero también su legitimidad y lo que constituye su formidable fuerza de atracción para los pueblos. Si perdemos esta fuerza, nuestra sociedad se derrumba. Es decir que la democracia está llamada a hacer de la educación su prioridad. Está llamada a la pedagogía. A replantearse su historia y sus aportes, a dar cuenta de inventiva incansable en este campo. Habrá que pensar en ello en este regreso a clases, para que nuestros niños aprendan pacientemente la virtud del debate democrático y para que las creencias llenas de odio y las reacciones identitarias no vengan a barrer con toda esperanza ni en la escuela ni en ningún otro lugar.

\section{Un nuevo paradigma para la formación inicial y continua de los profesores: "hacerse actor de coherencia"}

1. Primer principio: nunca perder de vista los objetivos. Lo que vale la pena enseñar es lo que une y lo que libera.

2. Segundo principio: recorrer sin parar la cadena, en los dos sentidos, desde los objetivos hasta las prácticas. Encarnar los objetivos en las prácticas. Referir las prácticas a los objetivos, ya que la coherencia entre los objetivos y las prácticas nunca se da de entrada, hay que construirla sin parar.

3. Tercer principio: cuestionarse siempre para inventar situaciones sobre la operación mental por suscitar y sobre las condiciones de su emergencia. Entre el objetivo y la situación, la operación mental es una hipótesis operacional que permite la inventiva.

4. Cuarto principio: alternar permanentemente las situaciones en las que se esté en posición de profesor y las situaciones en las que se esté en posición de "enseñado". Ponerse en posición de centración/decentración para interiorizar la exigencia de la inteligibilidad, comprender las resistencias y aprender a superarlas.

5. Quinto principio: identificar las competencias adquiridas en este proceso, formalizarlas, confrontarlas con los marcos de referencia, ya que saber que se sabe es mucho más que saber...

\section{Sexto principio: recordar sin cesar los objetivos} con el fin de ser "factor de coherencia".

El problema de nuestra democracia, el problema de nosotros, los adultos, desde el punto de vista de las generaciones que vienen, es el de cumplir con su palabra. ¿Cómo podemos pedirles a los jóvenes que cumplan su palabra, que sean leales, fieles a sus compromisos, cuando en nuestras reacciones familiares, educativas y políticas oscilamos todo el tiempo entre el autoritarismo y el laxismo? ¿Cómo podemos pedirles a los jóvenes que se construyan cuando nos cuesta hacer que nuestros objetivos sean coherentes con nuestras prácticas, a tal punto de precipitarse en la dilatación de las dos? 
No creamos, amigos míos, que la coherencia entre los objetivos y las prácticas es algo fácil, no creamos que es algo que nos sería otorgado simplemente por nuestra buena voluntad. En efecto, muchas de nuestras instituciones anuncian objetivos generales y generosos mientras que reproducen prácticas al menor costo. Así, nos satisfacemos en el plano narcisista con un despliegue de buenas intenciones, mientras que nos satisfacemos en el plano económico con la reproducción de lo idéntico.

Entre los objetivos y las prácticas, es necesario que construyamos la coherencia obstinadamente. La coherencia es, tal vez, lo más subversivo que existe hoy. Hay que volcarse sin cesar hacia aquellos y aquellas que anuncian objetivos formidables y preguntarles firmemente: "Pero, ¿por qué no hace lo que dice?" Por supuesto, lo que vale para la política, vale también para nosotros a diario, de tanto que nos cuesta hacer que nuestras convicciones sean coherentes con nuestras prácticas.

Recorrer sin parar la cadena, en los dos sentidos, preguntarse permanentemente cómo nuestros objetivos pueden encarnarse en nuestras prácticas y cómo nuestras prácticas reflejan nuestros objetivos; cuestionar permanentemente, en este ir y venir incansable y arduo, la coherencia de nuestro compromiso es - creo- una de las condiciones fundamentales para dar credibilidad a toda forma de democracia, sea en el seno de cada una de nuestras asociaciones, en el seno de cada una de nuestras instituciones, en el seno de cada clase, en el seno de cada familia. Y no creamos que esto es fácil: estamos siempre tentados a buscar excusas y escapatorias, estamos siempre tentados a yuxtaponer bellos discursos y prácticas mediocres, estamos siempre tentados a refugiarnos en las circunvoluciones de justificaciones coyunturales. Después no hará falta creer que las prácticas están contenidas en los objetivos como las nueces en sus cáscaras: las prácticas se dan en el marco de un trabajo de invención; requieren a la vez imaginación y obstinación, reflexión y confrontación, en cada grado, en cada nivel. Eso es lo que crea a todas las grandes figuras de la educación, aquello a lo que estamos llamados hoy. Ese trabajo que hace de nosotros "actores de coherencia" es fundacional, esencial. Para nosotros mismos, por supuesto, pero también para las generaciones jóvenes, porque si no encarnamos este esfuerzo de coherencia inventiva, de fidelidad a nuestros ideales, perdemos credibilidad, no podemos exigir nada, dejamos triunfar al mismo tiempo a la hipocresía y a la desesperanza, al arribismo y al populismo.

\section{A manera de conclusión: reconstruir la "mesa redonda"}

Escuchemos lo que dice Marcel Mauss a este respecto, en la conclusión de su bello ensayo sobre el don:

Para empezar, primero hubo que deponer las lanzas. Así es como el clan, la tribu, los pueblos, supieron -y mañana las naciones y los individuos deberán saberlo- cómo enfrentarse sin masacrase y darse sin sacrificarse los unos a los otros. He allí uno de los secretos permanentes de su sabiduría. [...] Las Crónicas de Arturo cuentan cómo el rey, con la ayuda de un carpintero de Cornouailles, creó esta maravilla de la corte: la mesa milagrosa alrededor de la cual los caballeros no se batieron más. [...] No hace falta ir a buscar más lejos el bien y la felicidad. Allí están, en la paz compartida y el trabajo armonioso, en común y en solitario, alternadamente. Está en la riqueza acumulada y luego redistribuida en el respeto mutuo y la generosidad recíproca que la educación enseña. (Mauss, 1957, p. 279).

Sin embargo, ya no tenemos a mano al carpintero de Cornouailles. Lo buscamos desesperadamente y Hannah Arendt nos dice hasta qué punto es difícil de encontrar, cuando en La condición humana nos explica que "el mundo común, que nos junta y no obstante impide que caigamos uno sobre otro, ha desaparecido progresivamente". Precisa:

\begin{abstract}
El mundo que hay entre nosotros no tiene el poder de reunirnos, de ligarnos, ni de separarnos. Extraña situación que evoca una sesión de espiritismo en la cual los participantes, víctimas de un pase mágico, vieran de pronto desaparecer la mesa, con lo cual las personas sentadas las unas en frente de las otras no estarían ya separadas, pero tampoco entrelazadas por nada tangible. (Arendt, 2002, p. 127).
\end{abstract}

$\mathrm{Y}$, efectivamente, ya no tenemos la mesa. Ya no hay para ligarnos ni separarnos, para estructurar nuestros intercambios, para invitarnos a deponer nuestras lanzas en la entrada... deponer nuestras lanzas, entrar en lo "simbólico", el symbolon — "sin jabalina"—; es decir, en la palabra, en la palabra argumentada, la palabra que respeta al otro y lo compromete a construir el mundo juntos. La palabra que nos permite, alrededor de la mesa, no precipitarnos los unos sobre los otros hasta la destrucción recíproca, sino reconocer participamos de "lo común" y que podemos perseguir nuestros intercambios para prolongarlos $y$ hacer juntos un mundo mejor.

Entonces, a falta del retorno del carpintero de Cornualles, ¿ ¿de qué disponemos para construir esta mesa? De los profesores y de la educación. De la 
educación y de los profesores. Actores esenciales, para hoy y para mañana, de una democracia con rostro humano.

\section{Bibliografía}

Arendt, H. (2002). Condition de l'homme moderne. Paris: Pocket-Agora.

Buisson, F. (2016). Dictionnaire de pédagogie et d'instruction primaire. Paris: Édition, collection "Bouquins ", Robert Laffont. http://www.inrp.fr/edition-electronique/ lodel/dictionnaire-ferdinand-buisson/

Freinet, C. (1945). L'Éducateur. Paris: PUF.

Hameline, D. (1986). L'éducation, ses images et son propos. Paris: ESF.

Hameline, D. (2000). Courants et contre-courants dans la pédagogie contemporaine. Paris: ESF.

Arendt, H. (2002). Condition de l'homme moderne, Paris, Pocket-Agora.
Kant, I. (1781). Critique de la raison pure. [Traduit en 1968] Paris : Presses Universitaires de France.

Mauss, M. (1957). Sociologie et Anthropologie. Paris: Quadrige/PUF.

Meirieu, P. (1995). La pédagogie entre le dire et le faire. Paris: ESF.

Meirieu, P. (2013). Pédagogie : des lieux communs aux concepts clés. Paris: ESF.

Morin, E. et Kern, A. (2010). Terre-Patrie. Paris: Le Seuil-Points.

Nussbaum, M. (2011). Les émotions démocratiques - Comment former le citoyen $d u X X X^{e}$ siècle ? Paris : Climats.

Reboul, O. (1989). La philosophie de l'éducation. Paris: PUF.

Vygotsky, L. (1985). Le problème de l'enseignement et du développement mental à l'âge scolaire. In B. Schneuwly et J.-P. Bronckart, Vygotsky aujourd'hui (pp. 95-117). Neuchâtel et Paris: Delachaux et Nietslé. 